\title{
Imaging translation dynamics in live embryos reveals spatial heterogeneities
}

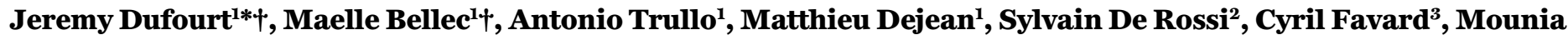 \\ Lagha ${ }^{1 *}$
}

${ }^{1}$ Institut de Génétique Moléculaire de Montpellier, University of Montpellier, CNRS-UMR 5535, Montpellier 34293 cedex 5, France. ²MRI, BioCampus Montpellier, CNRS, INSERM, University of Montpellier, Montpellier, France. ${ }^{3}$ Institut de Recherche en Infectiologie de Montpellier, CNRS UMR 9004, University of Montpellier, Montpellier 34293 cedex 5, France.

*Corresponding author. Email: jeremy.dufourt@igmm.cnrs.fr (J.D.); mounia.lagha@igmm.cnrs.fr (M.L.)

†These authors contributed equally to this work.

Much is known about the factors involved in the translation of mRNA into protein; however, this multi-step process has not been imaged in living multicellular organisms. Here, we deploy the SunTag method to visualize and quantify the timing, location, and kinetics of the translation of single mRNAs in living Drosophila embryos. By focusing on the translation of the conserved major epithelial-mesenchymal transition (EMT)-inducing transcription factor Twist, we identified spatial heterogeneity in mRNA translation efficiency and reveal the existence of translation factories, where clustered mRNAs are cotranslated preferentially at basal perinuclear regions. Observing the location and dynamics of mRNA translation in a living multicellular organism opens avenues for understanding gene regulation during development.

Over sixty years ago it was established that mRNA is translated to make protein. However, studies reveal that the level of a given mRNA and the amount of protein it encodes do not directly correlate (1). This lack of co-linearity may partially result, in part from differential translational regulation in subcellular compartments where mRNAs are targeted $(2,3)$. In order to quantitate and compare mRNA and nascent protein, methods are needed to visualize these molecules in vivo. Live imaging of mRNA has been possible since 1998 (4), but a similar method to image many cycles of translation was only established in 2016 in cultured cells (5-9), and has yet to be established in an intact developing organism. With its rapid development and the simple arrangement of nuclei in the syncytial blastoderm stage, Drosophila melanogaster embryo represents a model organism to image gene expression.

In order to visualize translation using a reporter transgene, we employed the SunTag system, whereby repetitions of an epitope (named suntag) are added to the protein of interest and are detected with a genetically encoded singlechain antibody (called scFv), fused to a fluorescent protein (10) (Fig. 1A). To implement the SunTag method in Drosophila embryos, we focused on the gene twist, which encodes a conserved transcriptional activator of the mesodermal program in metazoans (11). In Drosophila early embryos, this gene is expressed during the activation of the zygotic genome, in a specific ventral domain. We created a twi_suntag transgene (fig. S1F and supplementary text) that enables the labeling of Twi protein with 32 suntag repeats. In addition, we created scFv-fluorescent lines to detect suntag peptides (fig. S1, A to E; movie S8; and supplementary text). In the presence of the twi_suntag transgene and scFv-GFP detector protein, distinct spots were detected within the presumptive mesoderm of living embryos (figs. S1 and S2, movie S9, and supplementary text). However, twi_suntag expression appeared stochastically within this domain (fig. S1G; fig. S2, B, E, and F; and supplementary text).

Having demonstrated our ability to observe translation with a reporter transgene, we then monitored twi translational dynamics from its endogenous locus with a twi_suntag_CRISPR allele (fig. S3, A and B). By performing single molecule mRNA labeling (smFISH) with the simultaneous detection of native scFv-GFP, we could detect two populations of cytoplasmic mRNA molecules: those co-localizing with a bright GFP signal, i.e., $69 \% \pm 3 \%$ in n.c. 14 ( $\mathrm{n}=5$ embryos), presumably corresponding to mRNAs being translated, and those devoid of a GFP signal (Fig. 1B). Next, we questioned whether these bright scFv foci could be detected in living embryos with light sheet microscopy and found that twi translation was strongly induced during n.c.14 (Fig. 1C and movie S1), and was specific to the mesoderm. Bright but rare scFv-GFP foci appeared as early as n.c.12 and persisted during mitoses (fig. S3C).

To determine whether scFv-GFP spots correspond to nascent sites of translation, we imaged twi_suntag_CRISPR embryos injected with puromycin, a translation inhibitor. We did not observe scFv-GFP spots close to the injection site (fig. 
S3D and movie S10C). In order to observe nascent translation of single mRNA particles in live embryos, we engineered a twi_suntag_MS2_CRISPR and combined a scFv-mScarlet with a $M C P$-eGFP transgenic line (fig. S4 and supplementary text). For this dual cytoplasmic imaging, single mRNA molecules are labeled with an MS2 array, visualized using the coat protein of bacteriophage MS2, MCP fused to eGFP (12), while nascent proteins are labeled with the suntag peptides, recognized by the scFV antibody fused to mScarlet. Confocal imaging revealed distinct molecules of cytoplasmic mRNAs, with in some cases, a red scFv-mScarlet signal on top (fig. S4G and movie S2). This dual-color live imaging confirms the existence of two mRNA pools, with a subset of twi mRNA undergoing translation. It further shows these mRNA and nascent proteins move together (Fig. 1D and movie S3), revealing mRNAs in translation are not static.

By combining SunTag and MS2 labeling, it is possible to image transcription and translation and quantify their degree of correlation. In the case of $t w i$, the timing of translation is consistent with its mRNA production (fig. S5A). Live imaging of the twi_suntag_MS2_CRISPR reveals that transcription peaks in n.c.13 (Fig. 2, A and B) (13). Thus, the largest wave of mRNA production precedes the timing of the largest burst of twi translation (Fig. 2, C and D; fig. S4D; and movies $\mathrm{S} 1$ and $\mathrm{S} 12 \mathrm{~A}$ ). Further, the timing of twi translation is consistent with the timing of nuclear Twi protein emergence (fig. S5B).

To gain more insight into the dynamics of twi translation, we used the SunTag method to reveal translation kinetics (5, $7-9)$. We determined that Suntag-Twi fusion protein was fully translated (fig. S5C). Then, by correlating temporal intensity fluctuations of single spot scFv-GFP $(5,7-9)$, elongation and initiation rates were estimated to be in the order of $35 \mathrm{aa} / \mathrm{s}$ and $13 \mathrm{~s}$, respectively (fig. S5, D to H). These rates are probably upper estimates and do not reflect the important variability between mRNAs. Nonetheless, these rates lead to an overall translation efficiency of 7 ribosomes per mRNA (fig. S5I), consistent with ribosome profiling experiments (14). Collectively these data suggest the relatively late timing of twist translational activation could be partly compensated by its fast translation kinetics.

Using a transverse view of a developing embryo, the sites of translation in n.c.14 appeared much more prominent in the basal perinuclear region (i.e., toward the interior of the embryo), although translation was also observed in the apical perinuclear space (Fig. 3A, fig. S6A, and movies S4 and S12B). To further investigate this apparent spatial bias, we quantified the scFv-GFP signal in these two compartments (fig. S6, $\mathrm{B}$ and $\mathrm{C}$, and fig. S7). In contrast to earlier developmental stages, where translation is equivalent in the apical and basal cytoplasmic space, in n.c.14, the largest and brightest spots of twi translation appeared mainly in the basal cytoplasm. To estimate translation efficiency, we extracted the intensity of the scFv-GFP signal overlapping individual mRNA molecules (see materials and methods). We found that in the basal perinuclear space, a single molecule of mRNA is on average $50 \%$ more intense in scFv-GFP channel than a single molecule located apically, suggesting an enhanced efficiency of translation (Fig. 3, B and C). This bias is also observed with twi_suntag transgene (fig. S6, D and E). Collectively, these data demonstrate that translation efficiency of identical mRNA molecules depends on their subcellular localization. This spatial heterogeneity does not seem to rely on a differential distribution of ribosomes and might be supported by a higher basal availability of mitochondria (fig. S6, F and G, and movie S5).

Live imaging data revealed the existence of large $\mathrm{scFv}$ GFP foci, predominantly present in the basal cytoplasm. Simultaneous detection of mRNA and translation foci shows that these large size translation foci overlap large mRNA foci (Fig. $3 \mathrm{~B}$ and fig. S6D). To better characterize these large foci, we quantified mRNA densities and scFv-GFP signal. Although mRNA molecules were present along the entire depth of a cell volume, their intensity was clearly enhanced at the level of the basal perinuclear space (Fig. 4A), where they tend to assemble in clusters (fig. S8, A and B). These mRNA clusters were of varying sizes and were larger in the basal perinuclear cytoplasm (Fig. 4, A and B, and fig. S8, B and C). $94 \% \pm 3 \%$ of these mRNA clusters were engaged in translation $(n=4 \mathrm{em}-$ bryos). Thus, we consider them as translation factories, echoing what has been shown in mammalian cells $(7,15,16)$. Similar translation factories are observed with an ilp4-suntag transgenic reporter (fig. S9 and supplementary text).

Twi translation factories are distinct from germ plasm granules and p-bodies (fig. S10, A and B, and movie S15). Clustering of twi mRNA in the basal cytoplasm is also observed in wild-type as well as in twi hemizygous embryos, albeit with a reduced frequency suggesting that clustering partly depends on mRNA concentration (fig. S8D). Basal mRNA clustering is also detected for other mRNAs (fig. S11A). However, clustering of mRNAs is not a specific feature of the basal cytoplasm, as it is also observed apically (fig. S11, B and C) and largely documented for pair-rule genes $(17,18)$.

The site of twi mRNA major clustering might be, in part, dictated by the localization of its site of transcription (Fig. 4C; fig. S12, A and B; and movie S6). A preferential export of mRNA toward the basal cytoplasm would favor basal twi mRNA clustering which would be rapidly co-translated in factories. In the case of a nuclear protein like Twi, its translation in factories nearby the nuclear periphery could favor rapid nuclear import of newly formed proteins, as suggested by Twi protein stainings (fig. S12C).

twi translation occurs before complete cellularization. Consequently, its messenger ribonucleoproteins (mRNPs) 
could theoretically diffuse between neighboring pseudo-cells. To gain insight into twi mRNPs mobilities, we tracked twi_suntag_CRISPR mRNPs, in different cytoplasmic locations (Fig. 4D and movie S7). The trajectories and the mean square displacement (MSD) revealed clear distinct properties of apical versus basal particles (fig. S13 and supplementary text). Indeed, the diffusion coefficient of mRNPs is threetimes slower in the basal compartment (Fig. 4E). The sublinear growth of the MSD curves suggests sub-diffusive behavior in both compartments (fig. S11, C to G). Thus, we concluded that, in the basal perinuclear cytoplasm, $t w i$ translation sites diffuse slower due to their larger size.

By focusing on twi mRNAs as a paradigm for transcription factor encoding transcripts, we have uncovered fundamental features of translation in a living organism such as heterogeneity in translation efficiencies of identical mRNAs and the existence of translation factories. Local translation of multiple mRNAs could have several benefits. First it could favor the assembly of newly synthesized proteins in complexes. This is potentially the case for Twi known to homodimerize (19). Second, localized protein synthesis could favor fast delivery of newly formed protein to their destinations. Correlation between mRNA localization and protein function is well documented (2). The SunTag method now allows bridging the gap between mRNA and protein localization. In the case of $t w i$, we propose that local and enhanced translation close to the nuclear envelope favor rapid nuclear import of neosynthesized Twi protein. This might be general to other transcription factors, as proposed for pair-rule proteins (18).

Third, clustering of mRNA and their co-translation restricts mRNPs diffusion capacities. In the context of a syncytial embryo, this property could be exploited to limit the diffusion and allow spatial precision in cell fate decisions. As cellularization proceeds with an apico-basal directionality, apical anchoring of mRNAs, represents an optimal strategy to limit diffusion. However, translation dynamics of these apical mRNAs remain to be demonstrated. In contrast, for mRNAs located basally, in a compartment, where short-range diffusion lasts for a relatively long period of time, we propose that clustering and rapid local translation restrict mRNPs diffusion capacities. Thus, precision in the establishment of developmental patterns cannot only be attributed to precision in transcriptional activation. We anticipate that our approaches will pave the way toward hitherto inaccessible translation modalities during development and differentiation.

\section{REFERENCES AND NOTES}

1. K. Becker, A. Bluhm, N. Casas-Vila, N. Dinges, M. Dejung, S. Sayols, C. Kreutz, J.-Y. Roignant, F. Butter, S. Legewie, Quantifying post-transcriptional regulation in the development of Drosophila melanogaster. Nat. Commun. 9, 4970 (2018), doi:10.1038/s41467-018-07455-9 Medline

2. E. Lécuyer, H. Yoshida, N. Parthasarathy, C. Alm, T. Babak, T. Cerovina, T. R. Hughes, P. Tomancak, H. M. Krause, Global analysis of mRNA localization reveals a prominent role in organizing cellular architecture and function. Cell 131, 174-187 (2007). doi:10.1016/i.cell.2007.08.003 Medline

3. A. Chin, E. Lécuyer, RNA localization: Making its way to the center stage. Biochim. Biophys. Acta Gen. Subj. 1861, 2956-2970 (2017). doi:10.1016/i.bbagen.2017.06.011 Medline

4. E. Bertrand, P. Chartrand, M. Schaefer, S. M. Shenoy, R. H. Singer, R. M. Long, Localization of ASH1 mRNA particles in living yeast. Mol. Cell 2, 437-445 (1998). doi:10.1016/S1097-2765(00)80143-4 Medline

5. X. Yan, T. A. Hoek, R. D. Vale, M. E. Tanenbaum, Dynamics of Translation of Single mRNA Molecules In Vivo. Cell 165, 976-989 (2016). doi:10.1016/j.cell.2016.04.034 Medline

6. T. Morisaki, K. Lyon, K. F. DeLuca, J. G. DeLuca, B. P. English, Z. Zhang, L. D. Lavis, J. B. Grimm, S. Viswanathan, L. L. Looger, T. Lionnet, T. J. Stasevich, Real-time quantification of single RNA translation dynamics in living cells. Science 352 , 1425-1429 (2016). doi:10.1126/science.aaf0899 Medline

7. X. Pichon, A. Bastide, A. Safieddine, R. Chouaib, A. Samacoits, E. Basyuk, M. Peter, F. Mueller, E. Bertrand, Visualization of single endogenous polysomes reveals the dynamics of translation in live human cells. J. Cell Biol. 214, 769-781 (2016). doi:10.1083/jacb.201605024 Medline

8. B. Wu, C. Eliscovich, Y. J. Yoon, R. H. Singer, Translation dynamics of single mRNAs in live cells and neurons. Science 352, 1430-1435 (2016) doi:10.1126/science.aaf1084 Medline

9. C. Wang, B. Han, R. Zhou, X. Zhuang, Real-Time Imaging of Translation on Single mRNA Transcripts in Live Cells. Cell 165, 990-1001 (2016). doi:10.1016/j.cell.2016.04.040 Medline

10. M. E. Tanenbaum, L. A. Gilbert, L. S. Qi, J. S. Weissman, R. D. Vale, A proteintagging system for signal amplification in gene expression and fluorescence imaging. Cell 159, 635-646 (2014). doi:10.1016/i.cell.2014.09.039 Medline

11. T. Sandmann, C. Girardot, M. Brehme, W. Tongprasit, V. Stolc, E. E. M. Furlong, A core transcriptional network for early mesoderm development in Drosophila melanogaster. Genes Dev. 21, 436-449 (2007). doi:10.1101/gad.1509007 Medline

12. K. Tantale, F. Mueller, A. Kozulic-Pirher, A. Lesne, J.-M. Victor, M.-C. Robert, S. Capozi, R. Chouaib, V. Bäcker, J. Mateos-Langerak, X. Darzacq, C. Zimmer, E. Basyuk, E. Bertrand, A single-molecule view of transcription reveals convoys of RNA polymerases and multi-scale bursting. Nat. Commun. 7, 12248 (2016). doi:10.1038/ncomms12248 Medline

13. J. E. Sandler, A. Stathopoulos, Quantitative Single-Embryo Profile of Drosophila Genome Activation and the Dorsal-Ventral Patterning Network. Genetics 202. 1575-1584 (2016). doi:10.1534/genetics.116.186783 Medline

14. S. W. Eichhorn, A. O. Subtelny, I. Kronja, J. C. Kwasnieski, T. L. Orr-Weaver, D. P. Bartel, mRNA poly(A)-tail changes specified by deadenylation broadly reshape translation in Drosophila oocytes and early embryos. eLife 5, e16955 (2016). doi:10.7554/eLife.16955 Medline

15. L. Chang, Y. Shav-Tal, T. Trcek, R. H. Singer, R. D. Goldman, Assembling an intermediate filament network by dynamic cotranslation. J. Cell Biol. 172, 747758 (2006). doi:10.1083/icb.200511033 Medline

16. R. Chouaib, A. Safieddine, X. Pichon, A. Imbert, O. S. Kwon, A. Samacoits, A.-M. Traboulsi, M.-C. Robert, N. Tsanov, E. Coleno, I. Poser, C. Zimmer, A. Hyman, H. Le Hir, K. Zibara, M. Peter, F. Mueller, T. Walter, E. Bertrand, A Dual Protein-mRNA Localization Screen Reveals Compartmentalized Translation and Widespread Cotranslational RNA Targeting. Dev. Cell 54, 773-791.e5 (2020). doi:10.1016/i.devcel.2020.07.010 Medline

17. I. Davis, D. Ish-Horowicz, Apical localization of pair-rule transcripts requires 3 sequences and limits protein diffusion in the Drosophila blastoderm embryo. Cell 67, 927-940 (1991). doi:10.1016/0092-8674(91)90366-7 Medline

18. S. L. Bullock, M. Stauber, A. Prell, J. R. Hughes, D. Ish-Horowicz, U. Schmidt-Ott, Differential cytoplasmic mRNA localisation adjusts pair-rule transcription factor activity to cytoarchitecture in dipteran evolution. Development 131, 4251-4261 (2004). doi:10.1242/dev.01289 Medline

19. I. Castanon, S. Von Stetina, J. Kass, M. K. Baylies, Dimerization partners determine the activity of the Twist bHLH protein during Drosophila mesoderm development. Development 128, 3145-3159 (2001). Medline

20. J. Dufourt, A. Trullo, J. Hunter, C. Fernandez, J. Lazaro, M. Dejean, L. Morales, S. Nait-Amer, K. N. Schulz, M. M. Harrison, C. Favard, O. Radulescu, M. Lagha, 
Temporal control of gene expression by the pioneer factor Zelda through transient interactions in hubs. Nat. Commun. 9, 5194 (2018). doi:10.1038/s41467-01807613-z Medline

21. T. Ferraro, E. Esposito, L. Mancini, S. Ng, T. Lucas, M. Coppey, N. Dostatni, A. M. Walczak, M. Levine, M. Lagha, Transcriptional Memory in the Drosophila Embryo. Curr. Biol. 26, 212-218 (2016). doi:10.1016/i.cub.2015.11.058 Medline

22. K. J. Venken, Y. He, R. A. Hoskins, H. J. Bellen, P[acman]: A BAC transgenic platform for targeted insertion of large DNA fragments in $D$. melanogaster. Science 314, 1747-1751 (2006). doi:10.1126/science.1134426 Medline

23. S. C. Little, M. Tikhonov, T. Gregor, Precise developmental gene expression arises from globally stochastic transcriptional activity. Cell 154, 789-800 (2013). doi:10.1016/i.cell.2013.07.025 Medline

24. H. J. Motulsky, R. E. Brown, Detecting outliers when fitting data with nonlinear regression - a new method based on robust nonlinear regression and the false discovery rate. BMC Bioinformatics 7, 123 (2006). doi:10.1186/1471-2105-7-123 Medline

25. B. Rappaz, P. W. Wiseman, Image Correlation Spectroscopy for Measurements of Particle Densities and Colocalization. Curr. Protoc. Cell Biol. 59, 4.27.1-4.27.15 (2013). doi:10.1002/0471143030.cb0427s59

26. N. O. Petersen, Scanning fluorescence correlation spectroscopy. I. Theory and simulation of aggregation measurements. Biophys. J. 49, 809-815 (1986). doi:10.1016/S0006-3495(86)83709-2 Medline

27. U. Krzic, S. Gunther, T. E. Saunders, S. J. Streichan, L. Hufnagel, Multiview lightsheet microscope for rapid in toto imaging. Nat. Methods 9, 730-733 (2012). doi:10.1038/nmeth.2064 Medline

28. J. Schindelin, I. Arganda-Carreras, E. Frise, V. Kaynig, M. Longair, T. Pietzsch, S. Preibisch, C. Rueden, S. Saalfeld, B. Schmid, J.-Y. Tinevez, D. J. White, V. Hartenstein, K. Eliceiri, P. Tomancak, A. Cardona, Fiji: An open-source platform for biological-image analysis. Nat. Methods 9, 676-682 (2012). doi:10.1038/nmeth.2019 Medline

29. F. de Chaumont, S. Dallongeville, N. Chenouard, N. Hervé, S. Pop, T. Provoost, V. Meas-Yedid, P. Pankajakshan, T. Lecomte, Y. Le Montagner, T. Lagache, A. Dufour, J.-C. Olivo-Marin, Icy: An open bioimage informatics platform for extended reproducible research. Nat. Methods 9, 690-696 (2012). doi:10.1038/nmeth.2075 Medline

30. P. Müller, P. Schwille, T. Weidemann, PyCorrFit-generic data evaluation for fluorescence correlation spectroscopy. Bioinformatics 30, 2532-2533 (2014). doi:10.1093/bioinformatics/btu328 Medline

31. J. Widengren, Ü. Mets, R. Rigler, Photodynamic properties of green fluorescent proteins investigated by fluorescence correlation spectroscopy. Chem. Phys. 250, 171-186 (1999). doi:10.1016/S0301-0104(99)00255-4

32. Z. Liu, W. R. Legant, B.-C. Chen, L. Li, J. B. Grimm, L. D. Lavis, E. Betzig, R. Tjian, 3D imaging of Sox2 enhancer clusters in embryonic stem cells. elife 3, e04236 (2014). doi:10.7554/eLife.04236 Medline

33. A. Sergé, N. Bertaux, H. Rigneault, D. Marguet, Dynamic multiple-target tracing to probe spatiotemporal cartography of cell membranes. Nat. Methods 5, 687-694 (2008). doi:10.1038/nmeth.1233 Medline

34. D. Normanno, L. Boudarène, C. Dugast-Darzacq, J. Chen, C. Richter, F. Proux, 0. Bénichou, R. Voituriez, X. Darzacq, M. Dahan, Probing the target search of DNAbinding proteins in mammalian cells using TetR as model searcher. Nat. Commun. 6, 7357 (2015). doi:10.1038/ncomms8357 Medline

35. N. Tarantino, J.-Y. Tinevez, E. F. Crowell, B. Boisson, R. Henriques, M. Mhlanga, F. Agou, A. Israël, E. Laplantine, TNF and IL-1 exhibit distinct ubiquitin requirements for inducing NEMO-IKK supramolecular structures. J. Cell Biol. 204, 231-245 (2014). doi:10.1083/jcb.201307172 Medline

36. H. Qian, M. P. Sheetz, E. L. Elson, Single particle tracking. Analysis of diffusion and flow in two-dimensional systems. Biophys. J. 60, 910-921 (1991). doi:10.1016/S0006-3495(91)82125-7 Medline

37. A. Stathopoulos, M. Van Drenth, A. Erives, M. Markstein, M. Levine, Whole-genome analysis of dorsal-ventral patterning in the Drosophila embryo. Cell 111, 687-701 (2002). doi:10.1016/S0092-8674(02)01087-5 Medline

\section{ACKNOWLEDGMENTS}

We are grateful to E. Bertrand, R. Zinzen, I. Izzedin, P. Lasko, T. Hurd and X. Pichon for sharing flies, reagents and software. We thank C. Desplan, J. Chubb, R. Bordonne, F. Besse, T.E. Saunders, J. Dejardin and V.L. Pimmett, for their critical reading of the manuscript. We acknowledge L. Bellec, H. Lenden and M. Goussard for technical assistance. We acknowledge the MRI imaging facility (France-Biolmaging). Funding: MB is a recipient of an FRM fellowship. This work was supported by the ERC SyncDev starting grant and a HFSP-CDA grant to ML. $\mathrm{ML}, \mathrm{JD}$ and CF are sponsored by the CNRS. SDR is sponsored by the INSERM. Author contributions: ML conceived the project. ML and JD designed the experiments. JD, MB and MD performed experiments. AT developed software. $\mathrm{CF}, \mathrm{MB}$ and JD performed kinetic analysis. SDR and JD performed MuViSPIM imaging. JD, ML, AT, MB and CF analyzed the data. JD, ML, and MB interpreted the results. MB performed artwork. ML wrote the manuscript with help from JD and MB. All authors discussed, approved and reviewed the manuscript. Competing interests: Authors declare that they have no competing interests. Data and materials availability: All data are available in the main text and/or the supplementary materials and fly stocks will be deposited at the Bloomington Stock Center.

\section{SUPPLEMENTARY MATERIALS}

science.sciencemag.org/cgi/content/full/science.abc3483/DC1

Materials and Methods

Supplementary Text

Supplementary Sequences

Figs. S1 to S14

Table S1

References (20-37)

MDAR Reproducibility Checklist

Movies S1 to S15

29 April 2020; accepted 13 April 2021

Published online 29 April 2021

10.1126/science.abc3483 
A

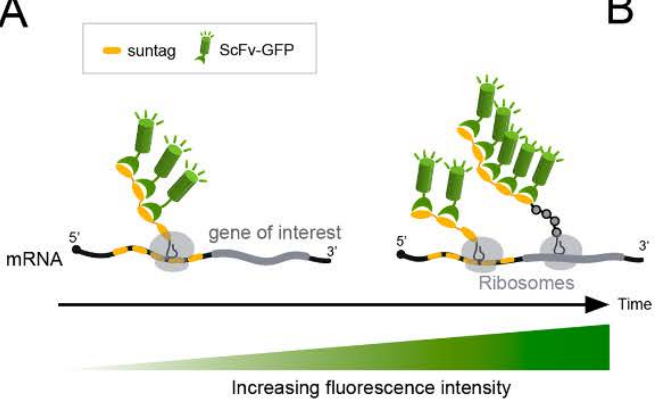

Increasing fluorescence intensity

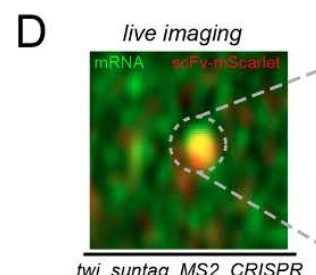

B

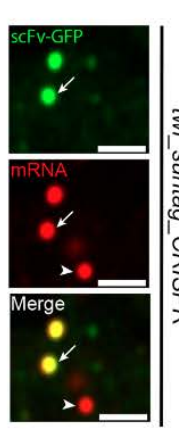

C

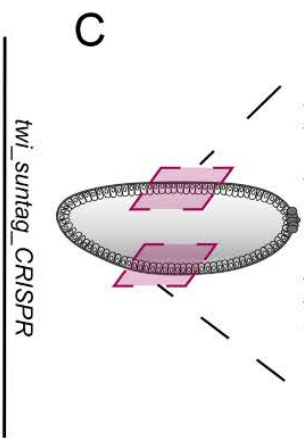

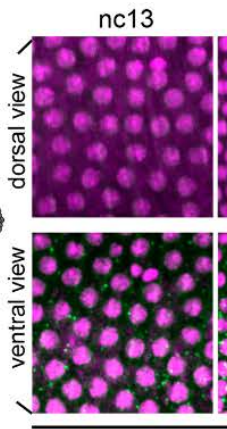

early nc14

mid nc14
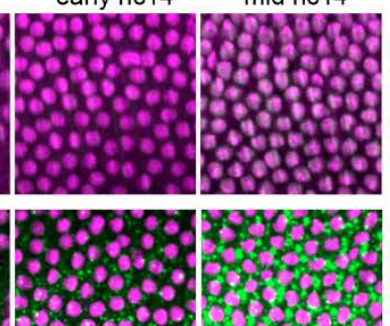

twi_suntag_CRISPR

Fig. 1. Imaging translation of mRNAs in Drosophila embryos. (A) Principle of the SunTag system. Repetitions of suntag epitopes are added to the protein of interest and are detected with a single-chain antibody (scFv) fused to sfGFP. (B) Zoomed confocal images of n.c.14 twi_suntag_CRISPR Drosophila embryos expressing scFv-GFP (green) stained with suntag probes (red), exhibiting two groups of mRNA molecules, co-localizing with scFvGFP signal (arrows) and not co-localizing with a GFP signal (arrowheads). Scale bars $1 \mu \mathrm{m}$. (C) Live imaging of a His2Av-mRFP/+;scFv-GFP-NLS/+>twi_suntag_CRISPR/+ embryo by MuViSPIM (Images from movie S1). (D) Spatiotemporal tracking of mRNA and translation signal from an MCP-eGFP/+; scFv-mScarletNLS/+>twi_suntag_MS2_CRISPR/+, embryo (Image from movie S3). 


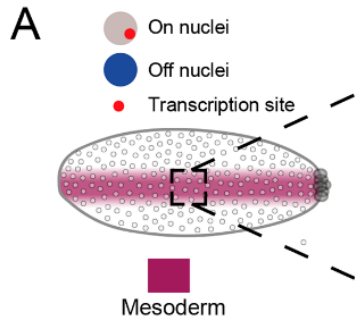

C
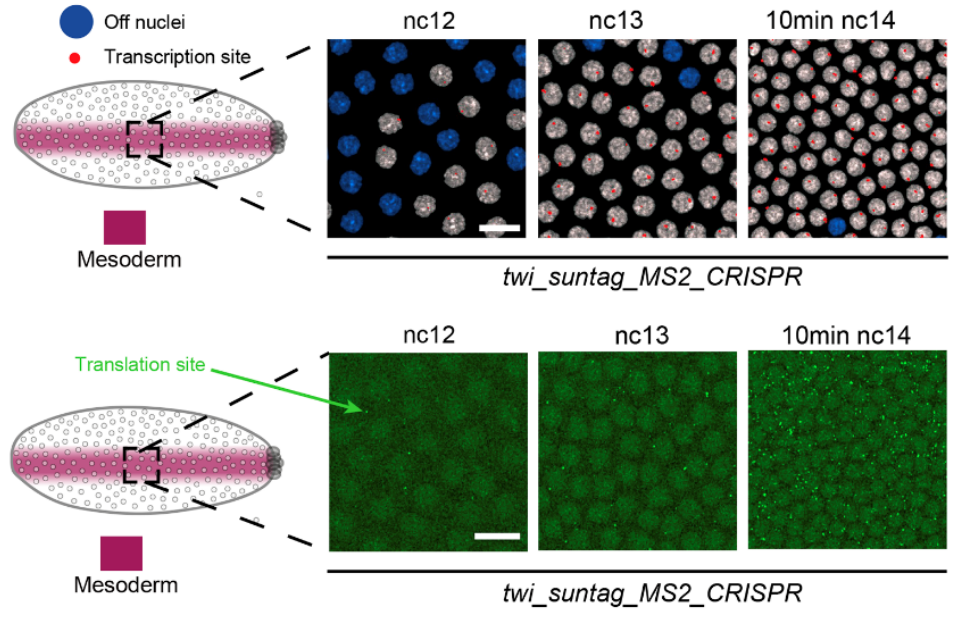

B

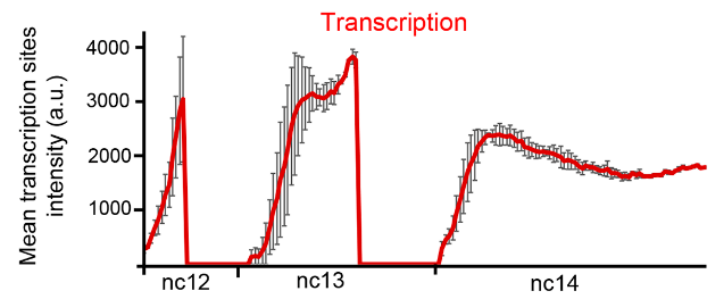

D

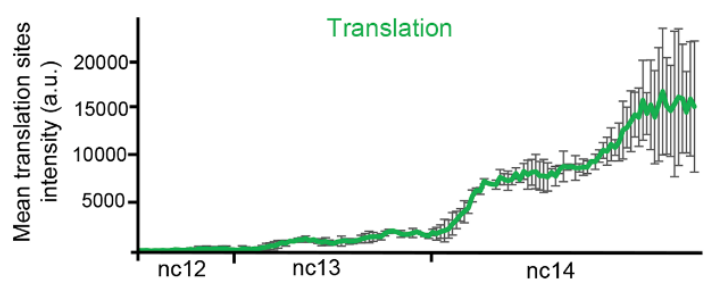

Fig. 2. Dynamics of transcription and translation. (A and $B$ ) Live imaging of a MCP-eGFP-His2AvmRFP>twi_suntag_MS2_CRISPR/+ embryo showing (A) transcription sites (TS) in red, and quantification of TS intensities in (B) ( $\mathrm{n}=2$ movies). Scale bars $10 \mu \mathrm{m}$. (C and $\mathrm{D})$ Live imaging of a scFvGFP/+>twi_suntag_MS2_CRISPR/+ embryo and quantification of translation site intensities over time $(\mathrm{n}=2$ movies). Scale bars $10 \mu \mathrm{m}$. 

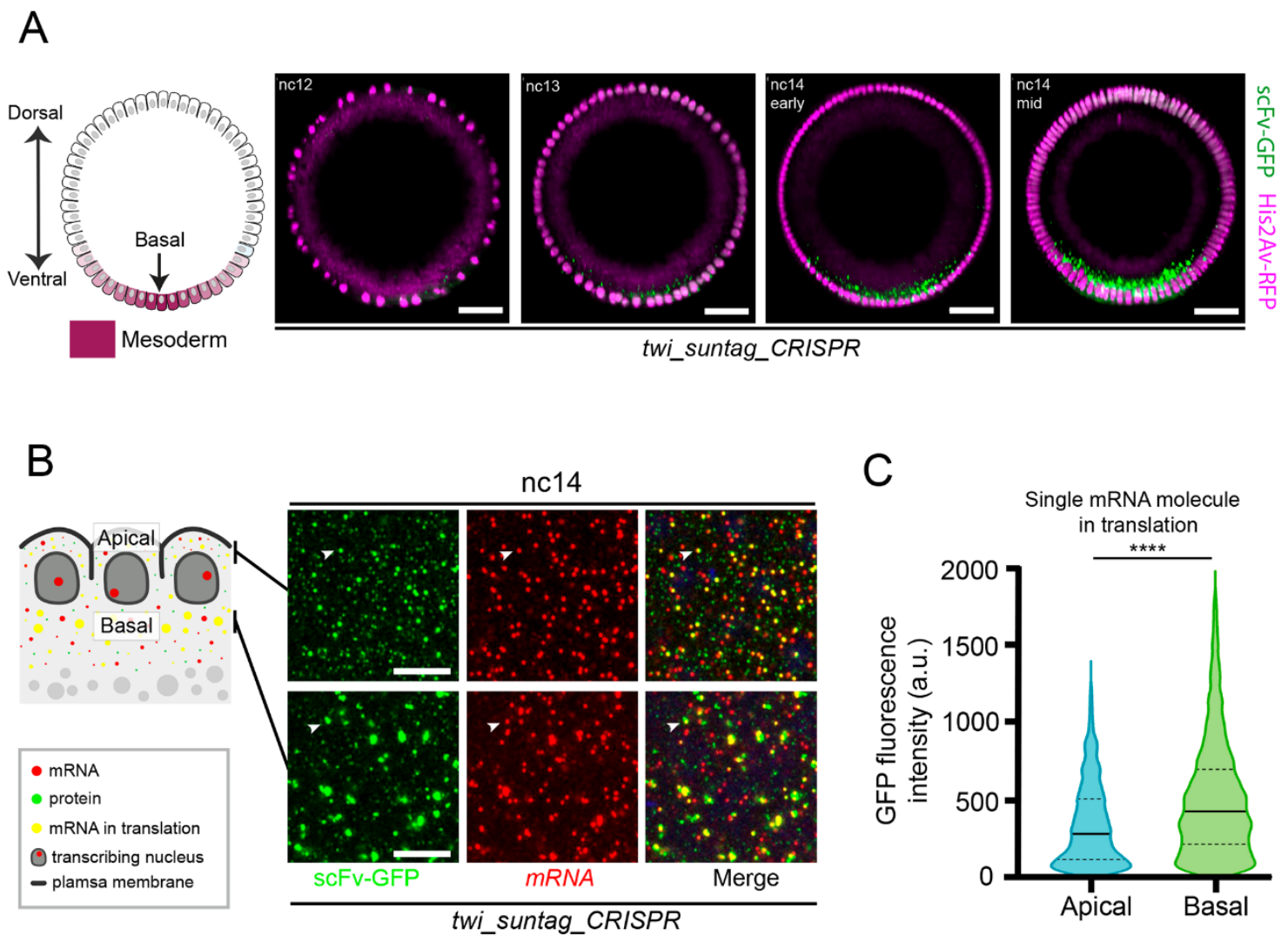

Fig. 3. Spatial heterogeneity of translation. (A) Live imaging of a His $2 A v-m R F P /+; S c F v$ GFP-NLS/+>twi_suntag_CRISPR/+ embryo (MuviSpim cross section) (Image from movie S4). scale bars, $30 \mu \mathrm{m}$. (B and C) Representative confocal image (apical and basal z-stacks shown separately) of an SCFv-GFP-NLS/+>twi_suntag_CRISPR/+ embryo expressing scFvGFP (green) labeled with suntag probes (red), scale bars $5 \mu \mathrm{m}(\mathrm{B})$ and quantification in (C), shown as a violin plot of the distribution of scFv-GFP intensities colocalizing with single mRNA molecules located apically $(n=2380$; blue) and basally $(n=4202$; green). two-tailed Welch's $t$ test, ${ }^{* * *} \mathrm{p}<0.0001$. 
A

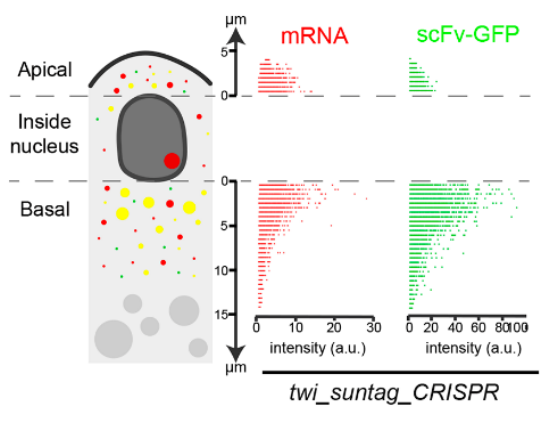

B

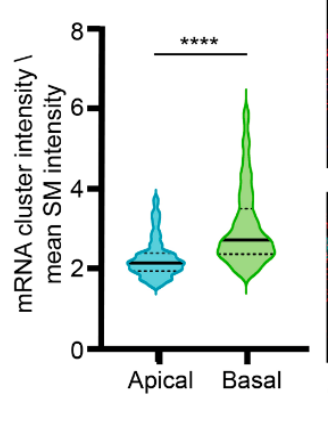

C

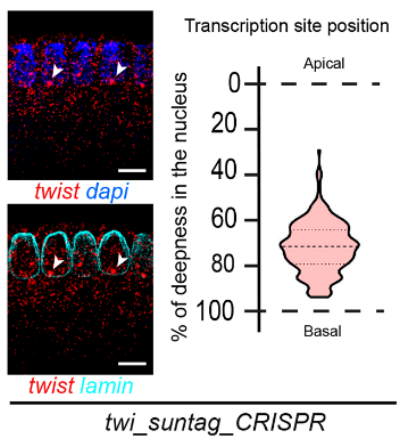

D

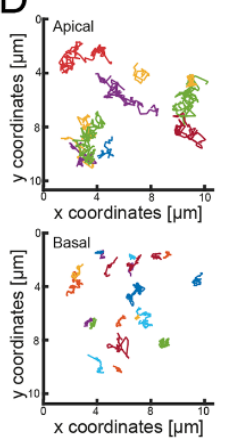

$E$

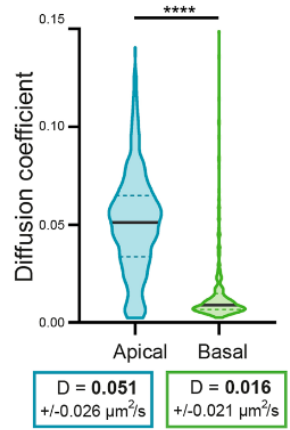

Fig. 4. Local translation of twi mRNA. (A) Intensity quantification of scFv-GFP-NLS/+>twi_suntag_CRISPR/+ embryos labeled with suntag probes showing mRNA spots (red) and scFv-GFP spots (green) in $0.5 \mu \mathrm{m}$ spaced Z-planes of four n.c.14 embryos. (B) Distribution of the intensity of mRNA clusters in apical $(n=523$, blue) and basal $(n=1384$, green), normalized by the mean intensity of a single mRNA molecule. Two-tailed Welch's $t$ test, **** $<<0.0001$. (C) Sagittal views of twi_suntag_CRISPR/+ embryos labeled with suntag probes, anti-lamin and DAPI, (scale bars $5 \mu \mathrm{m}$ ) and corresponding quantification of TS positions along the apico-basal nuclear axis. (D and E) Single particle tracking on scFv-GFP-NLS/+>twi_suntag_CRISPR/+ embryos. (D) Examples of colorcoded translation foci trajectories. (E) Violin plots of the estimated diffusion coefficient distributions of apical and basal particles. Two-tailed Welch's $t$ test, ${ }^{* * *} p<0.0001$. 